\title{
Uniformidade de distribuição de água em pivô central, com a utilização da técnica TDR na superfície e no interior do solo
}

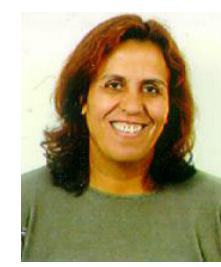

\author{
Tânia R.I. Rodrigues ${ }^{1}$, Helbert S. Batista ${ }^{2}$, Jane M. de Carvalho ${ }^{2}$, Alexandre O. Gonçalves ${ }^{2}$ \& Edson E. Matsura ${ }^{3}$ \\ 1 FEAGRI/UNICAMP. CP 6011, CEP 13081-970, Campinas, SP. Fone: (19) 788-1029 (Foto) \\ 2 FEAGRI/UNICAMP. CP 6011, CEP 13081-970, Campinas, SP. Fone: (19) 788-1029 \\ 3 FEAGRI/UNICAMP. E-mail: matsura@agr.unicamp.br
}

Protocolo $048-05 / 05 / 2000$

\begin{abstract}
Resumo: A avaliação da uniformidade de distribuição de água de um sistema de irrigação é feita acima da superfície do solo. Tendo em vista a importância da uniformidade no interior do solo quando submetido a uma irrigação, desenvolveu-se o presente trabalho com o objetivo de se avaliar a uniformidade de distribuição de água, tanto acima como no interior do solo, através dos coeficientes CUD e CUC, com um pivô central operando em condições de campo. Acima da superfície, a uniformidade foi obtida seguindo-se a metodologia da Associação Brasileira de Normas Técnicas (ABNT) e se alocando duas linhas de coletores com abertura de $3^{\circ}$, a partir do ponto inicial do pivô. Propôs-se, para obtenção da uniformidade no interior do solo, a utilização da umidade determinada pelo TDR (Reflectometria no Domínio do Tempo) visto que é uma técnica simples e produz resultados imediatos. Os resultados encontrados demonstraram que a uniformidade no interior do solo foi maior que na superfície, ficando acima da faixa recomendada, mostrando que a redistribuição da água no interior do solo promove maior uniformidade de umidade no perfil do solo que a encontrada na lâmina aplicada; portanto, no dimensionamento econômico de sistemas de irrigação, os coeficientes de uniformidade obtidos no interior do solo devem ser considerados.
\end{abstract}

Palavras-chave: irrigação, redistribuição de água, CUC, CUD

\section{Uniformity of water distribution in a central pivot with the use of the Time Domain Reflectometry technique on surface and soil}

\begin{abstract}
The evaluation of the uniformity of water distribution in an irrigation system is done above the surface of the soil. The main objective of this work was to evaluate the uniformity of water distribution in a central pivot under field conditions, through the use of the CUD and CUC coefficients. On the surface, the uniformity was obtained with the use of the ABNT methodology, therefore placing two collector lines displaced three degrees from the initial pivot position. In order to obtain the uniformity in the soil, the water content was determined with the TDR (Time Domain Reflectometry), which is a simple and fast technique. The results demonstrated that uniformity in the soil was greater than on the surface and above the recommended range. The water arrangement in the soil promotes that condition. Therefore in the economic design of irrigation systems, the uniformity coefficients in the soil should be considered.
\end{abstract}

Key words: irrigation, water arrangement, CUC, CUD

\section{INTRODUÇÃO}

Dentre os métodos de irrigação, o de aspersão por pivô central tem-se expandido de forma mais significativa, pelas seguintes razões: boa uniformidade de distribuição de água, quando bem planejado; fácil controle da lâmina d'água aplicada; grande versatilidade para as diversas condições de topografia e tipos de solo; menor dispêndio de mão-de-obra e maior divulgação por parte de fabricantes e vendedores (Bernardo, 1989).
A análise e a avaliação de um sistema de irrigação do tipo pivô central requerem, entre outras informações, o conhecimento da distribuição da água aplicada, uma vez que uma baixa uniformidade de distribuição pode levar a resultados insatisfatórios, reduzindo a eficiência de aplicação.

Seginer (1979) demonstrou que o aumento na uniformidade de distribuição de água pode aumentar o rendimento das culturas irrigadas e reduzir as perdas por percolação, resultando em benefícios econômicos e ambientais.

Segundo Heermann et al. (1992) o aumento da uniformidade de distribuição de água requer investimentos na melhoria do 
sistema, em manutenção e mão-de-obra para o manejo racional da irrigação.

Muitos pesquisadores têm avaliado os diversos sistemas de irrigação pela uniformidade de distribuição de água na superfície do solo. Segundo Rocha et al. (1998) a avaliação desses parâmetros apenas por medidas na superfície do solo, desconsiderando-se a redistribuição da água no seu perfil, que é tridimensional e de grande importância nas relações água-solo-planta, pode subestimar a sua eficiência de funcionamento.

Hart \& Reynolds (1965) estudaram, através de um modelo matemático, a influência de diversas variáveis de irrigação na redistribuição de água dentro do solo, e concluíram que a avaliação de sistemas de aspersão pelas medidas de superfície subestima a sua uniformidade sendo, portanto, inadequada, se o objetivo do projeto é resultar no sistema mais econômico. Já Letey (1985) relata as limitações das previsões de produtividade em função da uniformidade de aplicação da água de irrigação medida na superfície do solo. Neste mesmo trabalho, o autor recomenda determinações de uniformidade, com base em pontos localizados a distâncias compatíveis com a escala do sistema radicular da cultura em consideração.

São poucas as pesquisas encontradas na literatura com relação à uniformidade de distribuição da água no perfil do solo; dentre as mais recentes, pode-se citar Rezende (1992), Rezende et al. (1998) e Rocha et al. (1998).

Para obtenção dos coeficientes acima da superfície do solo, utilizam-se lâminas de água coletadas em pluviômetros e, para o interior do solo, empregam-se dados de umidade, normalmente obtidos por meio de amostragem gravimétrica, tensiômetros, sonda de nêutrons ou, ainda, da utilização da técnica eletromagnética através do TDR (Reflectometria no Domínio do Tempo).

O equipamento TDR mede a umidade do solo através da técnica de microondas. Segundo Tommaselli (1997) esta técnica se baseia no efeito do conteúdo de água no solo sobre a velocidade de propagação de um pulso eletromagnético e/ou no tempo que um pulso emitido leva para retornar ao sistema, após percorrer uma sonda instalada no solo, dada à grande diferença entre as constantes dielétricas da água e as dos componentes da matriz do solo. São necessários, basicamente, um testador de cabos e um sensor com hastes metálicas ligados por um cabo coaxial. Esta técnica reúne algumas vantagens importantes, como o tempo de resposta das leituras, podendo obter valores de umidade em tempo real; característica não destrutiva; garantia de segurança do operador no seu manuseio; não utiliza radiação ionizante e tem portabilidade e facilidade de acoplamento a dispositivos coletores de dados, porém apresenta a desvantagem de depender do tipo de solo (mineral e orgânico), do teor de materiais magnéticos do solo e de ter a calibração um pouco trabalhosa.

Mediante os fatos citados, nota-se que em qualquer sistema de irrigação a determinação dos coeficientes de uniformidade para superfície é bastante corriqueira, mas pouco explorada é a sua determinação para o interior do solo, através da técnica da TDR no monitoramento da umidade do solo.

Com base neste contexto, o presente trabalho teve como objetivo avaliar os coeficientes de uniformidade de distribuição da água na superfície e no interior do solo, de um sistema de irrigação tipo pivô central, utilizando-se os coeficientes de uniformidade de Christiansen (CUC) e o de uniformidade de distribuição (CUD) a partir do uso da TDR, tendo como base a utilização da equação de Topp et al. (1980) dita como universal para a medida indireta da umidade no solo, através da constante dielétrica $(\mathrm{ka})$.

\section{MATERIAL E MÉTODOS}

O estudo foi realizado no Instituto Agronômico de Campinas, onde se avaliou o desempenho de um equipamento de irrigação por aspersão tipo pivô central, da marca Valley, modelo 4865-8000-VL/5-542, fabricado pela Valmont com $313 \mathrm{~m}$ de raio, 5 torres acionadoras, balanço de $25 \mathrm{~m}$ e canhão no final da linha com alcance de $13 \mathrm{~m}$; distribuídos ao longo da lateral encontravam-se 130 aspersores da marca Seninger, com tubos de descida.

O ensaio foi realizado no dia 29 de outubro de 1999 e a cultura instalada na área irrigada era um milho híbrido C905 - PC2, plantado nos dias 2 e 3 de setembro de 1999.

Enfim, a avaliação do sistema baseou-se nas normas da ABNT (1985) constituindo-se de duas linhas de coletores com ângulo de abertura de $3^{\circ}$, a partir do ponto do pivô (Figura 1).

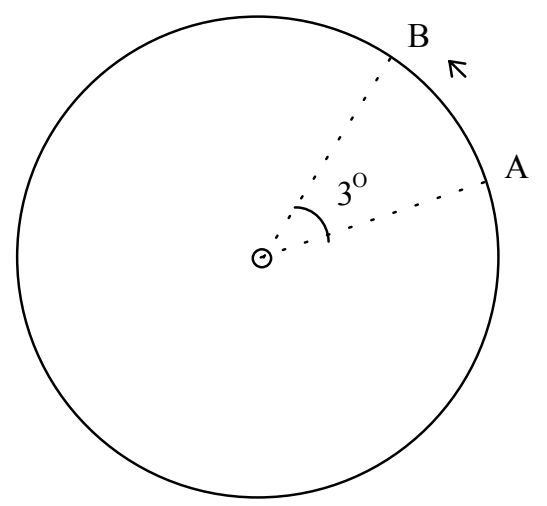

Figura 1. Linhas de coletores A e B alocadas na área irrigada

O primeiro coletor foi colocado a 13,5 m do ponto do pivô, sendo que os demais foram espaçados de $5 \mathrm{em} 5 \mathrm{~m}$, totalizando 61 coletores até o final de cada linha; cada coletor foi colocado em hastes metálicas a aproximadamente $50 \mathrm{~cm}$ da superfície do solo.

O pivô iniciou o funcionamento às 12:00 h, no sentido de rotação anti-horário e, após um período de estabilização de seu funcionamento, iniciou-se a avaliação propriamente dita. O percentímetro foi regulado a $50 \%$ da velocidade, aplicando-se uma lâmina média de $3,6 \mathrm{~mm}$, segundo dados de projeto.

No ponto do pivô foi medida a pressão através de um manômetro tipo Bourdon, mantida constante a $6,5 \mathrm{kgf} \mathrm{cm}^{-2}$ durante todo o ensaio, enquanto o volume aplicado foi monitorado através de um hidrômetro da marca Turbobar, localizado próximo ao ponto do pivô, durante $1 \mathrm{~h}$ permitindo, assim, a obtenção da vazão. Esta mesma vazão foi calculada empiricamente, através da Eq. (1):

$$
\mathrm{Q}=\frac{\pi \mathrm{R}^{2} \overline{\mathrm{z}}}{\mathrm{T}} 10^{-3}
$$


donde:

$$
\begin{array}{ll}
\mathrm{Q} & \text { - vazão total do sistema, } \mathrm{m}^{3} \mathrm{~h}^{-1} \\
\pi & -3,1415 \ldots . . \\
\mathrm{R} & \text { - raio irrigado, } \mathrm{m} \\
\overline{\mathrm{Z}} & \text { - lâmina média, } \mathrm{mm} \\
\mathrm{T} & \text { - tempo total de giro, } \mathrm{h}
\end{array}
$$

O tempo total de giro foi obtido a partir da velocidade da última torre, a qual foi calculada tomando-se uma largura de $20 \mathrm{~m}$, marcando-se o tempo gasto para percorrer esta distância.

O ensaio foi encerrado após a passagem completa do pivô central pelas duas linhas laterais de coletores, enquanto para a determinação dos coeficientes de uniformidade (CUC e CUD) foram utilizadas as equações:

$$
\mathrm{CUC}=100\left[1-\frac{\sum\left|\mathrm{z}_{\mathrm{i}}-\overline{\mathrm{z}}\right|}{\overline{\mathrm{z}} \mathrm{N}}\right]
$$

donde:

CUC - coeficiente de uniformidade de Christiansen, \%

$\bar{Z}_{i} \quad$ - lâmina coletada no pluviômetro i, $\mathrm{mm}$

z $\quad$ - lâmina média, $\mathrm{mm}$

$\mathrm{N} \quad$ - número de coletores

$$
\mathrm{CUD}=\frac{\overline{\mathrm{z}}_{(25)}}{\overline{\mathrm{Z}}} 100
$$

sendo:

CUD - coeficiente de uniformidade de distribuição segundo Davis (1966)

$\overline{\mathrm{z}}_{(25)}$ - lâmina média ponderada das menores precipitações correspondes a $25 \%$ da área, $\mathrm{mm}$

$\bar{Z} \quad$ - lâmina média, $\mathrm{mm}$

Os coeficientes de uniformidade acima da superfície, foram obtidos utilizando-se as lâminas médias precipitadas nas linhas de coletores A e B, os quais foram ponderadas de acordo com a distância do coletor ao centro do pivô.

Para obtenção dos coeficientes abaixo da superfície, utilizaram-se nas equações de CUC e CUD, valores de umidades médias obtidas após a irrigação, conforme os seguintes procedimentos: i) em cada linha de coletores, e com a utilização do TDR foram obtidos quatro valores de ka ao redor de cada coletor, a uma profundidade de $15 \mathrm{~cm}$; ii) fez-se a média de ka entre os quatro pontos medidos em cada coletor; iii) realizou-se a média de ka, entre as duas linhas de coletores na mesma posição; iv) com os valores médios de ka obteve-se a umidade média utilizando-se a equação de Topp et al. (1980) descrita abaixo:

$\mathrm{q}=\left(-5,3 \cdot 10^{-2}\right)+\left(2,92 \cdot 10^{-2} \cdot \mathrm{ka}\right)-\left(5,5 \cdot 10^{-4} \cdot \mathrm{ka}^{2}\right)+\left(4,3 \cdot 10^{-6} \cdot \mathrm{ka}^{3}\right)$

tal que:

$\theta \quad$ - umidade volumétrica, $\%$

ka - constante dielétrica

\section{RESULTADOS E DISCUSSÃO}

A partir da avaliação do sistema de irrigação, foi possível obter-se medidas e valores importantes que caracterizam o funcionamento do equipamento de irrigação e seu desempenho (Tabela 1).

Tabela 1. Características e condições do ensaio de avaliação

\begin{tabular}{lr}
\hline Velocidade da última torre $\left(\mathrm{m} \mathrm{h}^{-1}\right)$ & 185,0 \\
Tempo de giro $(\mathrm{h})$ & 10,6 \\
Pressão no centro do pivô $\left(\mathrm{kgf} \mathrm{cm}^{-2}\right)$ & 6,5 \\
Vazão de projeto $\left(\mathrm{m}^{3} \mathrm{~h}^{-1}\right)$ & 123,1 \\
Vazão medida no hidrômetro $\left(\mathrm{m}^{3} \mathrm{~h}^{-1}\right)$ & 99,0 \\
Vazão calculada $\left(\mathrm{m}^{3} \mathrm{~h}^{-1}\right)$ & 97,6 \\
\hline
\end{tabular}

De acordo com os dados do projeto com o relê a $100 \%$ da velocidade, o tempo de giro seria de aproximadamente $6 \mathrm{~h}$; nesta avaliação, usando-se $50 \%$ da velocidade, constatou-se que o tempo de giro do pivô foi de $10,6 \mathrm{~h}$ sendo que, pela indicação do painel, seria de $12 \mathrm{~h}$.

Pelo conteúdo da Tabela 1 observa-se que a vazão de projeto foi maior que a vazão do hidrômetro e a vazão calculada, devido ao fato de que o pivô operou a uma velocidade acima da projetada, diminuindo o tempo de giro e, conseqüentemente, aplicando uma lâmina menor. A diferença entre a vazão calculada e a medida no hidrômetro pode estar no fato da vazão do canhão não ter sido considerada na medição da vazão calculada.

O comportamento da lâmina coletada foi comparado com o da lâmina média projetada e o da coletada e, também, com a umidade após a irrigação, conforme se apresenta na Figura 2.

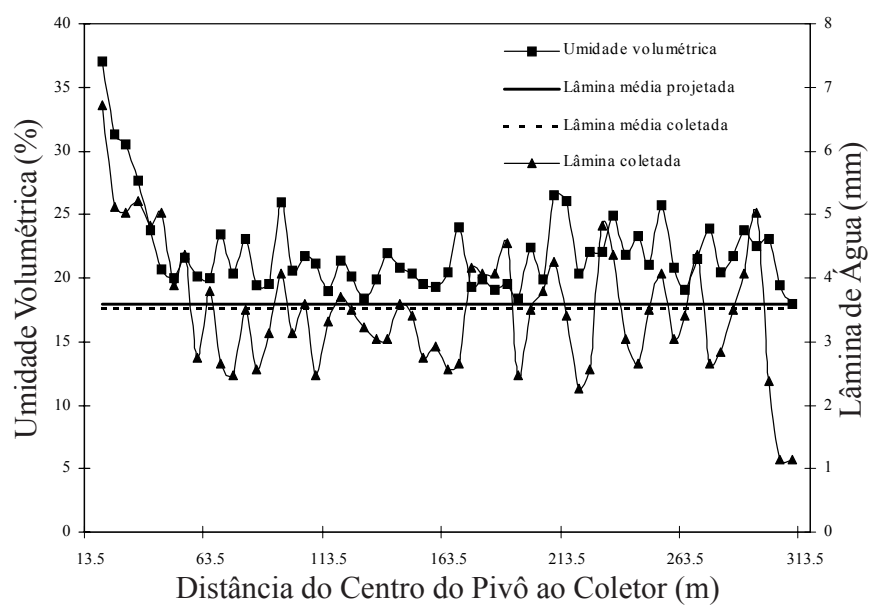

Figura 2. Valores de umidade volumétrica após a irrigação, lâmina média projetada, lâmina média coletada e valores de lâmina coletada

Constata-se, pelos resultados da Figura 2, que a lâmina média coletada foi inferior à lâmina média projetada, em que o equipamento aplicou menor quantidade de água que o projetado, podendo comprometer a efíciência de aplicação de água no solo. Observase, também, que, em geral, os valores de umidade apresentaram a mesma tendência dos valores de lâmina coletada, de maneira a permitir o uso do TDR na medição da uniformidade de distribuição de água no interior do solo, e que, para um ajuste polinomial entre 
a umidade e a lâmina coletada, observou-se o coeficiente de determinação de 0,54 , o que se considera significativo, sobretudo obtida em condições de campo (Figura 3 ).

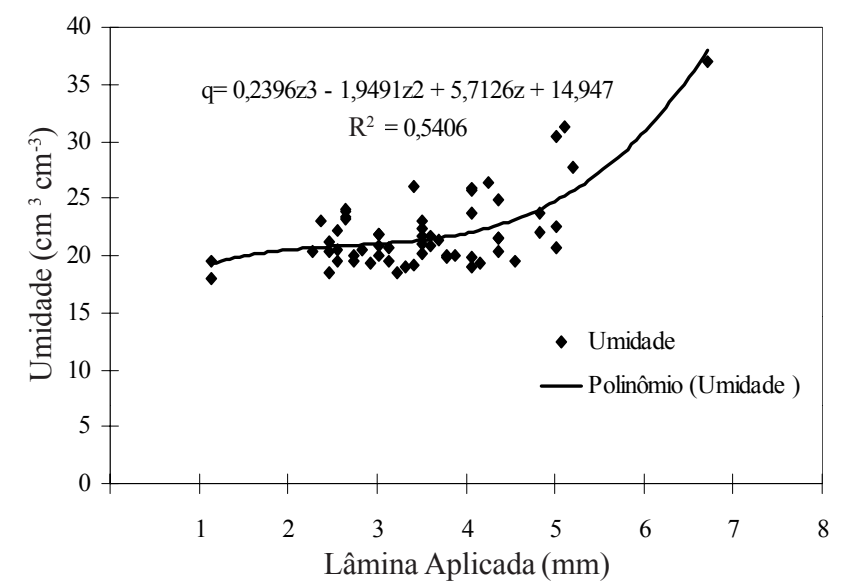

Figura 3. Correlação entre lâmina coletada e umidade, após irrigação

Na Figura 4 observa-se que a lâmina coletada nos coletores e a umidade obtida pelo TDR, apresentaram o mesmo comportamento, podendo-se observar, ainda, que em aproximadamente $75 \%$ da área irrigada se aplicou uma lâmina maior que a lâmina média, e 62,5\% da área irrigada apresentou umidade maior que a umidade média.

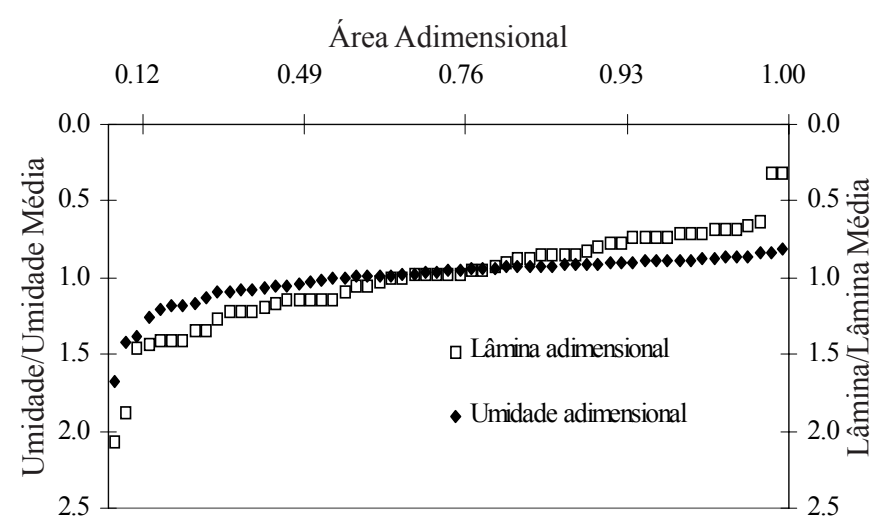

Figura 4. Distribuição da lâmina coletada e umidade, em relação à área irrigada adimensional

De acordo com as equações apresentadas, foram calculados os coeficientes CUC e CUD para as duas situações na superfície e no interior do solo, mostrados na Tabela 2.

Observa-se, pela Tabela 2, que o coeficiente de uniformidade de distribuição (CUD) foi menor que o coeficiente de uniformidade de Christiansen (CUC) tanto acima da superfície como no interior do solo. Este comportamento já era esperado, pelo fato de que o primeiro considera a média das $25 \%$ menores

Tabela 2. Valores dos coeficientes de uniformidade CUC e CUD, obtidos na superfície e no interior do solo

\begin{tabular}{|c|c|c|c|}
\hline \multicolumn{2}{|c|}{ Uniformidade na Superfície } & \multicolumn{2}{|c|}{ Uniformidade no Interior do Solo } \\
\hline CUC & CUD & CUC & CUD \\
\hline \multicolumn{4}{|c|}{ (\%) } \\
\hline 77,97 & 67,12 & 89,19 & 86,36 \\
\hline
\end{tabular}

lâminas coletadas e o de Christiansen pondera a média da lâmina coletada em todos os coletores. Zocoler (1999) recomenda valores entre 70 a $80 \%$ e 82 a $88 \%$ para CUD e CUC, respectivamente, para as culturas cujo sistema radicular explora, basicamente, os primeiros $40 \mathrm{~cm}$ do solo.

Rocha et al. (1998) e Rezende et al. (1998) pesquisando a distribuição sub e superficial da água em irrigação por aspersão convencional, constataram que o coeficiente de uniformidade de Christiansen (CUC) subsuperficial foi maior que na superfície, vindo de encontro com os resultados obtidos neste trabalho

Constata-se, ainda na Tabela 2, que na superfície os valores dos coeficientes ficaram abaixo do recomendado; entretanto, os valores obtidos no interior do solo estão dentro da faixa recomendada, uma vez que a redistribuição de água no interior do solo está diretamente relacionada com a produtividade da cultura, ao passo que a uniformidade na superfície está relacionada com a lâmina aplicada e diz respeito ao desempenho do equipamento.

\section{CONCLUSÕES}

1. A uniformidade de distribuição no interior do solo foi maior que na superfície, demonstrando que houve redistribuição da água e que o uso da técnica de medição de umidade no solo, através do equipamento TDR, foi adequada, podendo ser usada em trabalhos de campo desta natureza.

2. Para o dimensionamento econômico de sistemas, os coeficientes de uniformidade no interior do solo devem ser desconsiderados.

3. Em estudos futuros, seja feito um acompanhamento da redistribuição de água no solo, usando-se o TDR, a fim de que se possa conhecer a uniformidade da umidade para o correto manejo de irrigação.

\section{AGRADECIMENTOS}

Agradecemos ao Instituto Agronômico de Campinas, IAC, na pessoa do Prof. Dr. Flávio Arruda, que disponibilizou as instalações da Fazenda Santa Elisa para a realização deste trabalho, e ao Prof. Dr. José Geanini Peres, da Universidade Federal de São Carlos, Centro de Ciências Agrárias, pelo empréstimo do equipamento TDR.

\section{LITERATURA CITADA}

ABNT - Associação Brasileira De Normas Técnicas. Sistema de irrigação por aspersão pivô central, caracterização e desempenho - método de ensaio; $1^{\circ}$ Projeto de Normas. Rio de Janeiro, 1985. 22p. PN 12:02.08.005

Bernardo, S. Manual de irrigação. Viçosa, UFV. 1986. 456p.

Davis, J.R. Measuring water distribution from sprinklers.

Transaction of the ASAE, St. Joseph, v.9, n.1, p.94-7, 1966. Hart, W.E.; Reynolds, W.N. Analytical design of sprinkler systems.

Transactions of the ASAE, St. Joseph, v.8, n.10, p.83-9, 1965. 
Heermann, D.F.; Duke, H.R.; Serafim, A.M.; Dawson, L.L. Distribution functions to represent center-pivot water distribution. Transactions of the ASAE, St. Joseph, v.35, n.5, p.1465-1472, 1992.

Letey, J. Irrigation uniformity as related to optimum crop production - additional research is needed. Irrigation Science, v.6, p.253-263, 1985.

Rezende, R. Desempenho de um sistema de irrigação pivô central quanto à uniformidade de aplicação de água, abaixo e acima da superfície. Piracicaba: ESALQ, 1992. 88p. Dissertação Mestrado

Rezende, R.; Frizzone, J.A.; Gonçalves, A.C.A.; Freitas, P.S.L. de. Influência do espaçamento entre os aspersores na uniformidade da distribuição de água acima e abaixo da superfície do solo. In: Congresso Brasileiro de Engenharia Agrícola, 27, 1998, Poços de Caldas, MG. Anais... Poços de Caldas: Sociedade Brasileira de Engenharia Agrícola, 1998, p.130-2.
Rocha, E.M.M.; Mapurunga, S.M.S.; Costa, R.N.T.; Castro, P.T.; Aguiar, O.S. Uniformidade e eficiência de aplicação de água de irrigação por aspersão convencional na superfície e perfil do solo. In: Congresso Brasileiro de Engenharia Agrícola, 27, 1998, Poços de Caldas, MG. Anais... Poços de Caldas: Sociedade Brasileira de Engenharia Agrícola, 1998, p.286-8.

Seginer, I. Irrigation uniformity related to horizontal extent of root zone. Irrigation Science, v.1, p.89-96, 1979.

Tommaselli, J.T. Influência de algumas características do solo sobre a calibração de um aparelho de TDR (Time-Domain Reflectometry). Piracicaba: CENA, USP, 1997. 108p. Dissertação Mestrado

Topp, G.C.; Davis, J.L.; Annan, A P. Electromangetic determination of soil water content: Measurement in coaxial transmission lines. Washington: Water Resources Research, v.16, p.574-582. 1980.

Zocoler, J.L. Avaliação do desempenho de sistemas de irrigação In: Curso Capacitação em Agricultura Irrigada, 1999, Ilha Solteira, Anais... Ilha Solteira: UNESP/FEIS, 1999, 55p. 